\title{
Strategic use of environmental information
}

\author{
Geir B. Asheim*
}

March 23, 2010

\begin{abstract}
Strategic use of environmental information may have as consequence that a benevolent environmental agency will choose not to disclose information leading to reduced moral motivation. Thus, decision makers will not have access to such information, implying that they will not be able to adjust their decisions to available information on the state of the environment. In contrast, if the benevolent environmental agency instead bases its regulation on standard economic instruments, these instruments will incorporate all available information.
\end{abstract}

Keywords and Phrases: Environmental regulation, voluntary contributions, moral motivation, hard information

JEL Classification Numbers: D11, H41

${ }^{*}$ Department of Economics, University of Oslo, P.O. Box 1095 Blindern, 0317 Oslo, Norway (e-mail: g.b.asheim@econ.uio.no).

I thank Ted Bergstrom, Laura Grant, Cameron Hepburn, Steinar Holden, Tore Nilssen and Karine Nyborg for helpful suggestions, participants at the workshop "Voluntary Approaches to Environmental Protection" at the Bren School at UCSB and the CBE Conference on Behavioral Economics at WWU for comments, the Economics Department at UCSB for hospitality, and the Research Council of Norway through the project "Norms, green agents and environmental policy" for financial support. 


\section{Introduction}

The actions of consumers and firms may have external effects that lead to long-lasting harm to the natural environment. Current economic activity may also reduce the resource base and thus contribute to unsustainable development.

In order to prevent degradation of environmental quality and to ensure sustainable development, strategies that protect the natural environment and the livelihood of future generations must be implemented. In principle, such implementation can be achieved in two ways:

(i) Through environmental policies (i.e., by use of Pigovian taxes or other economic instruments) designed to make consumers and firms internalize the environmental costs of their actions, and endow them with bequest motives for the (natural and man-made) capital stocks they manage.

(ii) Through spread of information about the environmental consequences of the actions of consumers and firms, given that these agents have an underlying concern for the protection of the natural environment and the promotion of sustainable development.

The first method of implementation, e.g., through Pigovian taxes, is transparent, as it asks consumers and firms to pay the social costs of the external effects that their actions lead to, without interfering with decision-making in other respects.

On the other hand, information about the future development of the environment can be highly important for the emergence of moral motivation leading to environmentally friendly behavior and the formation of the bequest motives needed to ensure sustainable development. It is likely that individual decision makers will be more concerned about the environment if there is awareness about possible grave consequences that lack of such concern will lead to. Hence, one must be open for the possibility that individuals' behavior is influenced by information about the ag- 
gregate consequences of their actions. In addition, such information may impact the political feasibility of the environmental policies mentioned under (i).

The authorities, e.g., an environmental regulatory agency, can partially control the spread of information on the environmental consequences of actions taken by consumers and firms. E.g., the authorities may decide to extend funding of research where preliminary results seem to indicate that environmental consequences are serious and long-lasting, while terminating programs that downplay the need for environmental protection. Such selective support for research may even be given a seemingly plausible justification by appealing to the "precautionary principle".

Both the extent to which such information is generated and the manner in which it is presented can influence the actions consumers and firms wish to take, so that behavior is changed in an environmentally friendly direction. One may conjecture that a more aggressive informational policy may serve as a substitute for the kind of environmental instruments that economists normally suggest. In particular, by using information to influence people's environmental values and preferences, external effects may to some extent be internalized with less use of explicit instruments.

However, strategic use of information raises normative questions of the following type: How can authorities be under democratic control if they use information to manipulate the interests of their constituency? How can information be trusted if it is suspected (or known) that information is spread for strategic purposes?

In this paper, I will not address the former question, as I will assume that the environmental agency is benevolent, seeking to foster the interests of the individuals.

Rather, I will be concerned with the latter question. The present paper will present modeling designed to show that strategic use of environmental information may have as consequence that a benevolent environmental agency will choose not to disclose information leading to reduced moral motivation. This means that decision makers will not have access to such information, implying that they will not be able to adjust their decisions to available information on the state of the environment. 
In contrast, if the benevolent environmental agency instead bases its regulation on standard economic instruments, these instruments will incorporate all available information.

The model, which will be presented in Sections 2 and 3, will be based on the rationale for morally motivated behavior suggested by Brekke, Kverndokk and Nyborg (2003). While voluntary private provision of public goods (Bergstrom, Blume and Varian, 1986) will be negligible without social preferences, and the "warm glow" theory of giving (Andreoni, 1990) does not specify a connection between actual and ideal behavior, Brekke, Kverndokk and Nyborg's theory suggests that morally motivated behavior will depend on the behavior individuals believe will maximize social welfare. Therefore, information about the environmental consequences of their behavior will affect what they decide to do (Owen, Videras and Wu, 2008, present empirical support for this hypothesis). This in turn creates an incentive for a benevolent environmental agency to analyze information dissipation strategically.

Proposition 1 of Section 4 provides a condition under which the unique subgameperfect equilibrium has the property that information reducing the individuals' moral motivation will not be disclosed. Proposition 2 of Section 5 shows that such strategic use of environmental information may reduce welfare, compared to the situation where the agency commits to a full-disclosure policy. Section 5 also offers a short discussion of mechanisms which would enable the environmental agency to make such a commitment. The concluding Section 6 relates the present analysis to the literature on the crowding-out effect of economic instruments and summarizes the policy implications of the present results.

Kallbekken, Westskog and Mideksa (2010) also discuss whether spread of normative information is an efficient policy instrument in the presence of environmental externalities. Their interesting analysis complements the one presented here.

The analysis of the present paper is not limited to the case of an environmental agency that receives environmental information. It applies to any benevolent organi- 
zation that appeals to moral motivation in order to induce voluntary contributions. E.g., the results suggest that blood drives, seeking to generate voluntary donations, may conceal information that downplay the need for blood.

\section{Voluntary public good provision}

Consider an economy with $n$ individuals contributing to an environmental public good. Each of them chooses effort $e_{i}$, which is individual $i$ 's voluntary contribution. All individuals are identical, choosing $e_{i}$ from the unit interval. Individual $i$ 's private consumption is $1-e_{i}$ and the total provision of the public good is $\sum_{j=1}^{n} e_{j}$.

Each individual $i$ 's utility function is given by:

$$
u\left(e_{i}, e_{-i} ; e^{*}, \hat{e}\right)=1-e_{i}+e^{*} \ln \left(\sum_{j=1}^{n} e_{j}\right)-a\left|\hat{e}-e_{i}\right|,
$$

where $e_{-i}:=\left(e_{1}, \ldots, e_{i-1}, e_{i+1}, \ldots, e_{n}\right)$ is the effort profile of the other $n-1$ individuals, and $e^{*}>0, \hat{e}>0$ and $a>0$ are three parameters that determine the function $u$. Hence, utility is a quasi-logarithmic function of private consumption and the public good, with a linear loss function capturing the cost of deviating from the effort level $\hat{e}$ believed to be socially optimal by the individual and thus the effort level that drives his/her moral motivation. At the end of Section 4 it will be noted that the main result of this paper does not depend on this particular specification of the utility function.

Observe that $e^{*}$ is the effort level which maximizes $1-e+e^{*} \ln (n e)$. Hence, $e^{*}$ is the effort which, if made by all, would maximize the utility that each individual derives from private consumption and the public good. In their analysis, Brekke, Kverndokk and Nyborg (2003) assume that the individuals are correctly informed of the socially optimal effort, so that $\hat{e}=e^{*}$. In this case, $e^{*}$ is the effort which, if made by all, would maximize each individual's utility even when the moral motivation is 
taken into account:

$$
e^{*}=\arg \max _{e} u(e, \underbrace{(e, \ldots, e)}_{n-1 \text { times }}, e^{*}, e^{*}) .
$$

I will assume, like Brekke, Kverndokk and Nyborg (2003) do, that moral motivation is not sufficient to ensure that the actual effort of the individuals equals the socially optimal effort, $e^{*}$, even if their beliefs are correct. In the present model this assumption corresponds to the condition:

$$
a<1-\frac{1}{n} .
$$

This follows from the equilibrium analysis of Section 4 (cf. (4)) since each individual's effort in the unique Nash equilibrium is given by

$$
e=\frac{e^{*}}{(1-a) n}<e^{*}
$$

if (3) is combined with $\hat{e}=e^{*}$. Hence, then it is indeed the case that voluntary contributions fall short of the socially optimal level. Rather, the individuals will trade-off their moral obligation against their personal material gain from shirking.

\section{Hard information}

I will allow for the possibility that individuals are not perfectly informed of the socially optimal effort. Moreover, I will assume that the environmental agency receives signals that provide hard information concerning what the socially optimal effort is. Hard information, a concept introduced in information economics by Grossman (1981), Milgrom and Roberts (1986) and Tirole (1986), means that, if a signal has been received, the environmental agency can convey the informational content of the signal to the general public in a credible way. However, the environmental agency can conceal that a signal has been received, and it cannot credibly demonstrate that a signal has not been received.

In the present setting one can imagine that the agency is able to spread the informational content of a received signal through funding research and undertaking 
publicity campaigns, and that independent experts can testify that the publicized information is true if and only if the signal has actually been received. Thus, if the information is based on a received signal, individuals can look at the evidence and convince themselves that the agency has announced correct information.

In general, the environmental agency will receive signals that affect its belief of $e^{*}$ both positively and negatively. However, given the assumption that moral motivation is not sufficient to ensure that individuals make the socially optimal effort, it follows that a received signal affecting the belief of $e^{*}$ in a positive direction will be conveyed to the general public, since it will push effort in a welfare-enhancing direction. Therefore, only signals affecting the belief of $e^{*}$ in a negative direction are of interest for our analysis.

A parsimonious way of modeling an interesting situation is to assume that $e^{*}$ can take on only two values: $e_{h}^{*}$ with probability $p$ and $e_{\ell}^{*}$ with probability $1-p$, where $e_{\ell}^{*}<e_{h}^{*}$. One can think of $e_{h}^{*}$ and $e_{\ell}^{*}$ as two possible states of the environment, where effort to protect the environment is particulary needed if $e^{*}=e_{h}^{*}$. If the true value is $e_{\ell}^{*}$, then the environmental agency receives a signal which can, if the agency chooses to do so, be credibly conveyed to the general public. If the true value is $e_{h}^{*}$, then the environmental agency does not receive a signal and cannot credibly convey information to the general public.

Hence, the environmental agency is perfectly informed of $e^{*}$. The agency knows that $e^{*}$ equals $e_{h}^{*}$ if it does not receive the signal and equals $e_{\ell}^{*}$ if it does receive the signal. The strategy of the agency specifies what to do if a signal is received. The strategy $s(\in[0,1])$ signifies that the agency discloses the signal with probability $s$ if received. Hence, $s=1$ means to convey the informational content of the signal with probability 1 , and $s=0$ means to conceal receipt of the signal with probability 1. Say that the environmental agency uses information strategically if $s=0$. Figure 1 illustrates the game between the environmental agency and the individuals. 
$\langle$ Insert Figure 1 about here $\rangle$

Individuals have the prior belief that $e^{*}$ equals $e_{h}^{*}$ with probability $p$ and $e_{\ell}^{*}$ with probability $1-p$. If the signal is received and disclosed, then the individuals have received hard information demonstrating that $e^{*}$ equals $e_{\ell}^{*}$. If a signal is not conveyed, then the posterior belief of each individuals depends on his/her conjecture of the strategy of the agency. In equilibrium, the conjectures of the individuals will coincide. Denote by $r(\in[0,1])$ the conjecture of the individuals, meaning that the individuals believe that the agency discloses the signal with probability $r$ if received. Then the posterior belief $q(r)$ that the individuals assign to $e_{h}^{*}$ is given by:

$$
q(r)=\frac{p}{p+(1-p)(1-r)}
$$

In particular, if $r=1$ so that the individuals believe that the agency always discloses a received signal, then $q(r)=1$, entailing that the individuals are certain that the environmental state equals $e_{h}^{*}$ if a signal is not disclosed. On the other hand, if $r=0$ so that the individuals believe that the agency never discloses a received signal, then $q(r)=p$, entailing that the individuals' posterior belief is the same as their prior belief: $e^{*}$ equals $e_{h}^{*}$ with probability $p$ and $e_{\ell}^{*}$ with probability $1-p$.

\section{Equilibrium}

We face a dynamic game of incomplete information between the environmental agency and the $n$ individuals, as the agency but not the individuals are informed of whether a signal is received. Each individual has two information sets, corresponding to whether a signal has been disclosed or not. At both information sets, the equilibrium belief of each individual is derived from the strategies of the other players. Hence, it suffices to apply the concept of subgame-perfect equilibrium.

In order to determine the set of subgame-perfect equilibria, we first have to calculate the Nash equilibria in the subgame that the individuals play after the agency 
has disclosed a signal. In this subgame where the individuals have received hard information demonstrating that $e^{*}$ equals $e_{\ell}^{*}$, there exist a unique Nash equilibrium in which each individual $i$ chooses

$$
\begin{aligned}
e_{\ell} & =\arg \max _{e} u(e, \underbrace{\left(e_{\ell}, \ldots, e_{\ell}\right)}_{n-1 \text { times }} ; e_{\ell}^{*}, e_{\ell}^{*}) \\
& =\arg \max _{e}\left(1-e+e_{\ell}^{*} \ln \left(e+\frac{(n-1) e_{\ell}^{*}}{(1-a) n}\right)-a\left|e_{\ell}^{*}-e\right|\right)=\frac{e_{\ell}^{*}}{(1-a) n} .
\end{aligned}
$$

If, on the other hand, a signal is not conveyed, then the mutual best reply of the individuals depends on the conjecture $r$ of the individuals:

$$
\begin{aligned}
& e(r)=\arg \max _{e}( q(r) u(e, \underbrace{(e(r), \ldots, e(r))}_{n-1 \text { times }} ; e_{h}^{*}, e_{h}^{*}) \\
&\left.+(1-q(r)) u(e, \underbrace{(e(r), \ldots, e(r))}_{n-1 \text { times }} ; e_{\ell}^{*}, e_{\ell}^{*})\right) \\
&=\arg \max _{e}\left(q(r)\left(1-e+e_{h}^{*} \ln \left(e+\frac{(n-1) e^{*}(r)}{(1-a) n}\right)-a\left|e_{h}^{*}-e\right|\right)\right. \\
&\left.+(1-q(r))\left(1-e+e_{\ell}^{*} \ln \left(e+\frac{(n-1) e^{*}(r)}{(1-a) n}\right)-a\left|e_{\ell}^{*}-e\right|\right)\right) \\
&=\arg \max _{e}\left(1-e+e^{*}(r) \ln \left(e+\frac{(n-1) e^{*}(r)}{(1-a) n}\right)-a\left|e^{*}(r)-e\right|\right)=\frac{e^{*}(r)}{(1-a) n},
\end{aligned}
$$

where $e^{*}(r)=q(r) e_{h}^{*}+(1-q(r)) e_{\ell}^{*}$. This conclusion requires that

$$
a \leq 1-\frac{e_{h}^{*}}{n e_{\ell}^{*}}
$$

so that $e^{*}(r) /(1-a) n \leq e_{\ell}^{*}$ for all $r \in[0,1]$.

We then turn to the behavior of the environmental agency. The agency is benevolent, entailing that it wants to maximize the welfare of the individuals. In its evaluation of welfare, it will take into account both the actual state of the environment since this matters for the material well-being of the individuals, and the effect of the believed state of the environment since this drives the moral motivation of the individuals.

The environmental agency makes a decision only if a signal has been received, entailing that that the environmental state equals $e_{\ell}^{*}$. If it discloses the signal, then 
ex post welfare equals:

$$
u_{\ell}^{d}=u\left(e_{\ell},(\underbrace{\left(e_{\ell}, \ldots, e_{\ell}\right)}_{n-1 \text { times }}, e_{\ell}^{*}, e_{\ell}^{*})=1-\frac{e_{\ell}^{*}}{(1-a) n}+e_{\ell}^{*} \ln \frac{e_{\ell}^{*}}{1-a}-a \cdot e_{\ell}^{*}\left(1-\frac{1}{(1-a) n}\right) .\right.
$$

If, on the other hand, the signal is not conveyed, then ex post welfare depends on $r$ :

$$
\begin{aligned}
u_{\ell}(r)= & q(r) u(e(r), \underbrace{(e(r), \ldots, e(r))}_{n-1 \text { times }}, e_{\ell}^{*}, e_{h}^{*}) \\
& +(1-q(r)) u(e(r), \underbrace{(e(r), \ldots, e(r))}_{n-1 \text { times }}, e_{\ell}^{*}, e_{\ell}^{*}) \\
= & 1-\frac{e^{*}(r)}{(1-a) n}+e_{\ell}^{*} \ln \frac{e^{*}(r)}{1-a}-a \cdot e^{*}(r)\left(1-\frac{1}{(1-a) n}\right) .
\end{aligned}
$$

The choice of the environmental agency depends on whether $u_{\ell}(r)$ exceeds $u_{\ell}^{d}$. If $u_{\ell}(r)>u_{\ell}^{d}$ for all $r \in[0,1]$, then the agency will not disclose the signal for any conjecture of the individual. Hence, then the agency chooses $s=0$, and in equilibrium the conjecture of the individuals will be correct, so that $r=0$.

Proposition 1 Assume that $u_{\ell}(r)>u_{\ell}^{d}$ for all $r \in[0,1]$. Then there exists a unique subgame-perfect equilibrium in which the environmental regulatory agency uses information strategically, disclosing with probability 0 hard information showing that the environmental state is $e_{\ell}^{*}$.

Proof. Assume that the conjecture of the individuals equals $r$. The payoff of the agency conditional on the signal being received equals

$$
s u_{\ell}^{d}+(1-s) u_{\ell}(r) .
$$

Hence, if $u_{\ell}(r)>u_{\ell}^{d}$ for all $r \in[0,1]$, then the agency will choose $s=0$ independently of the conjecture $r$ of the individuals. Therefore, $r=0$, implying that the individuals will choose $e_{\ell}$ if a signal is disclosed, and $e(0)$ if a signal is not conveyed.

As the following corollary shows,

$$
a \leq \frac{e_{\ell}^{*}}{e_{h}^{*}}-\frac{1}{n}
$$

is a sufficient condition for $u_{\ell}(r)>u_{\ell}^{d}$ for all $r \in[0,1]$. 
Corollary 1 Assume that utility function is specified by (1) and satisfies (8). Then there exists a unique subgame-perfect equilibrium in which the environmental regulatory agency uses information strategically, disclosing with probability 0 hard information showing that the environmental state is $e_{\ell}^{*}$.

Proof. First, note that (8) implies (5), implying that $e(r)$ is indeed the mutual best reply for the individuals, given that their conjecture is $r$. We have that

$$
u_{\ell}(r)-u_{\ell}^{d}=-\frac{e^{*}(r)-e_{\ell}^{*}}{(1-a) n}+e_{\ell}^{*} \ln \frac{e^{*}(r)}{e_{\ell}^{*}}-a\left(e^{*}(r)-e_{\ell}^{*}\right)\left(1-\frac{1}{(1-a) n}\right) .
$$

By Proposition 1, it suffices to show that this expression is positive for all $r \in[0,1]$.

Define $f:\left[e_{\ell}^{*}, e_{h}^{*}\right] \rightarrow \mathbb{R}$ by

$$
f(x)=-\frac{x-e_{\ell}^{*}}{(1-a) n}+e_{\ell}^{*} \ln \frac{x}{e_{\ell}^{*}}-a\left(x-e_{\ell}^{*}\right)\left(1-\frac{1}{(1-a) n}\right) .
$$

Since

$$
u_{\ell}(r)-u_{\ell}^{d}=\int_{e_{\ell}^{*}}^{e^{*}(r)} f^{\prime}(x) d x,
$$

it is sufficient to show that $f^{\prime}(x)>0$ for all $x \in\left(e_{\ell}^{*}, e_{h}^{*}\right)$. This follows as

$$
f^{\prime}(x)=-\frac{1}{(1-a) n}+\frac{e_{\ell}^{*}}{x}-a \cdot\left(1-\frac{1}{(1-a) n}\right)=\frac{e_{\ell}^{*}}{x}-a-\frac{1}{n}>\frac{e_{\ell}^{*}}{e_{h}^{*}}-a-\frac{1}{n} \geq 0,
$$

where $x<e_{h}^{*}$ implies the strict inequality and (8) implies the weak inequality.

It is of interest to note the relationship between the weak inequality (8), the sufficient condition for strategic use environmental information, and the strict inequality (3), the condition under which moral motivation is not sufficient to ensure that the actual effort of the individuals equals the socially optimal level. Since

$$
\frac{e_{\ell}^{*}}{e_{h}^{*}}-\frac{1}{n}<1-\frac{1}{n}
$$

it follows that (8) implies (3). Hence, environmental information will be used strategically when moral motivation falls well short of what is needed for individuals to act according to their ideal, had they known it. 
The result of Proposition 1 does not depend on the particular utility function specified in (1). It is sufficient that the utility function $u\left(e_{i}, e_{-i} ; e^{*}, \hat{e}\right)$ satisfies $(2)$ and that $u_{\ell}(r)>u_{\ell}^{d}$ for all $r \in[0,1]$, where

$$
u_{\ell}^{d}=u\left(e_{\ell},(\underbrace{\left(e_{\ell}, \ldots, e_{\ell}\right)}_{n-1 \text { times }}, e_{\ell}^{*}, e_{\ell}^{*})\right.
$$

with $e_{\ell}=\arg \max _{e} u\left(e,\left(e_{\ell}, \ldots, e_{\ell}\right) ; e_{\ell}^{*}, e_{\ell}^{*}\right)$, and

$$
u_{\ell}(r)=q(r) u(e(r), \underbrace{(e(r), \ldots, e(r))}_{n-1 \text { times }}, e_{\ell}^{*}, e_{h}^{*})+(1-q(r)) u(e(r), \underbrace{(e(r), \ldots, e(r))}_{n-1 \text { times }}, e_{\ell}^{*}, e_{\ell}^{*})
$$

with $e(r)=\arg \max _{e}\left(q(r) u\left(e,(e(r), \ldots, e(r)) ; e_{h}^{*}, e_{h}^{*}\right)+(1-q(r)) u(e,(e(r), \ldots, e(r))\right.$;

$\left.\left.e_{\ell}^{*}, e_{\ell}^{*}\right)\right)$. The condition that $u_{\ell}(r)>u_{\ell}^{d}$ for all $r \in[0,1]$ entails that moral motivation is not sufficient to ensure that the actual effort of the individuals equals the socially optimal level, in that same way as (8) implies (3) in the special case that we have considered, of voluntary contribution to an environmental public good.

\section{$5 \quad$ Welfare analysis}

Under the assumptions of Proposition 1, the individuals do not obtain information that will enable them to update their prior belief since the agency uses and is believed to use information strategically. Hence, ex ante welfare equals

$$
u(0)=p u(e(0), \underbrace{(e(0), \ldots, e(0))}_{n-1 \text { times }}, e_{h}^{*}, e_{h}^{*})+(1-p) u(e(0), \underbrace{(e(0), \ldots, e(0))}_{n-1 \text { times }}, e_{\ell}^{*}, e_{\ell}^{*}) .
$$

If, on the other hand, the agency had always disclosed a received signal and the individuals had believed that a received signal would always be conveyed, then ex ante welfare would have been

$$
u(1)=p u(e(1), \underbrace{(e(1), \ldots, e(1))}_{n-1 \text { times }}, e_{h}^{*}, e_{h}^{*})+(1-p) u(e_{\ell}, \underbrace{\left(e_{\ell}, \ldots, e_{\ell}\right)}_{n-1 \text { times }}, e_{\ell}^{*}, e_{\ell}^{*}) .
$$

Proposition 2 Assume that utility function is specified by (1) and satisfies (8). Then ex ante welfare $u(0)$ in the unique subgame-perfect equilibrium with strategic 
use of environmental information is lower than ex ante welfare $u(1)$ in the case where the environmental agency had disclosed, and had been believed to disclose, with probability 1 hard information showing that the environmental state is $e_{\ell}^{*}$.

Proof. By using (6) and (7) and the definitions of $q(r)$ and $e^{*}(r)$, it follows that

$$
\begin{aligned}
& u(1)=1-\frac{e^{*}(0)}{(1-a) n}+p e_{h}^{*} \ln \frac{e_{h}^{*}}{1-a}+(1-p) e_{\ell}^{*} \ln \frac{e_{\ell}^{*}}{1-a}-a \cdot e^{*}(0)\left(1-\frac{1}{(1-a) n}\right), \\
& u(0)=1-\frac{e^{*}(0)}{(1-a) n}+e^{*}(0) \ln \frac{e^{*}(0)}{1-a}-a \cdot e^{*}(0)\left(1-\frac{1}{(1-a) n}\right) .
\end{aligned}
$$

Hence, using that $e^{*}(0)=p e_{h}^{*}+(1-p) e_{\ell}^{*}$, we obtain

$$
u(1)-u(0)=p e_{h}^{*} \ln e_{h}^{*}+(1-p) e_{\ell}^{*} \ln e_{\ell}^{*}-e^{*}(0) \ln e^{*}(0) .
$$

The fact that $e \ln e$ is a strictly convex function implies that $u(1)-u(0)>0$.

Proposition 2 entails that there is an informational cost — over and beyond the fact that moral motivation is insufficient to induce the optimal effort - of trying to internalize externalities without use of explicit economic instruments. Since information will be strategically concealed, even though the agency is benevolent with aligned interests, the individuals will not have access to all information, implying that they will not be able to adjust their decisions to available information on the state of the environment.

The above analysis models the interaction between the environmental agency and the individuals as a one-time interaction. If the agency and the individuals have an on-going relationship, then one must also consider reputational effects. In such a situation it is in the interest of the benevolent agency to build up a reputation for disclosing all information. In the case where the agency faces a small probability of detection if a received signal is concealed, it may choose to convey the signal due to the threat of losing its reputation as a trustworthy agency if it does not.

In line with the arguments for rules rather than discretion (Kydland and Prescott, 1977) in the context of monetary policy (see also Helm, Hepburn and Mash, 2003, 
for a discussion in the context of environmental policy), one may use the current analysis as a basis for proposing that an environmental agency, like a central bank, should be independent. Exploiting this analogy further (with reference to Rogoff, 1985), one may argue that the environmental agency should be headed by a director who cares less about the environmental public good than the individuals. In particular, suppose that the director's utility function is given by

$$
1-e+\frac{e^{*}}{(1-a) n} n e,
$$

where $e$ is the effort of each individual. Hence, compared to (1) he discounts the public good by the factor $1 /(1-a) n(<1$ by $(3))$ and does not care about the cost imposed on the individuals by not acting according to their moral motivation. Then the individuals will act optimally in the director's evaluation if they are fully informed. Thus, he will direct the agency to convey the signal whenever it is received.

Even though such institutional arrangements might remove the welfare costs associated with strategic use of environmental information, it will not remove the welfare costs that the insufficient moral motivation leads to. If instead the environmental agency has access to and is allowed to use perfect economic instruments, then optimal effort can be induced in each environmental state and similar informational problems will not be present. The agency will use instruments leading the optimal effort of $e_{h}^{*}$ if the signal is not received and to the optimal effort of $e_{\ell}^{*}$ if the signal is received. The individuals will realize that the agency has no reason to conceal information, given that there is no wedge between what individuals ideally should do and what they actually do. For the same reason, individuals will not feel a cost associated with not fulfilling their moral motivation. Hence, the ex ante welfare under the assumption that perfect economic instruments are used will be:

$$
p u(e_{h}^{*}, \underbrace{\left(e_{h}^{*}, \ldots, e_{h}^{*}\right)}_{n-1 \text { times }}, e_{h}^{*}, e_{h}^{*})+(1-p) u(e_{\ell}^{*}, \underbrace{\left(e_{\ell}^{*}, \ldots, e_{\ell}^{*}\right)}_{n-1 \text { times }}, e_{\ell}^{*}, e_{\ell}^{*}) .
$$

In this regulatory regime, optimal effort will be induced and all available information will be utilized. 


\section{Concluding remarks}

The results of the present paper relates to a line of literature that has developed during the last few years, relating to whether economic instruments crowd out other motivation for socially beneficial activity (see Nyborg and Rege, 2003, for a survey of this literature). Such theoretical analysis and related empirical evidence point to a cost of using economic instruments, since it weakens the intrinsic motivation that would otherwise guide people's actions. Does this mean that we should not apply economic instruments, and instead base environmental policy on the promotion of intrinsic motivation?

This paper has developed one argument against this view, namely that promotion of intrinsic motivation may deter disclosure of information on the environmental effects of economic behavior. This will in turn foster a public view that an environmental agency will only disclose information which proves that behavior has serious environmental effects, while not making available information showing the opposite. The analysis has shown that a benevolent environmental agency may indeed choose to behave in this manner if asked to base environmental policy on the promotion of intrinsic motivation, but will not do so when instructed to use economic instruments that can ensure efficient behavior.

Clear policy prescriptions follow from the model of the current paper. If perfect economic instruments are available, then they should be used, as they lead to optimal effort and ensure that all available environmental information is conveyed. If such instruments are not available and moral motivation is insufficient to induce optimal effort, then a benevolent environmental agency will ex post be tempted to use environmental information strategically which, however, from an ex ante perspective reduces welfare. Hence, the second-best policy prescription if perfect economic instruments cannot be used is to design institutional arrangements that will enable the environmental agency to maintain a reputation for disclosing all information. 


\section{References}

Andreoni, J. (1990), Impure altruism and donations to public goods: A theory of warm-glow giving, Economic Journal 100, 464-477.

Bergstrom, T., Blume, L. and Varian, H. (1986), On the private provision of public goods, Journal of Public Economics 29, 25-49.

Brekke, K.A., Kverndokk, S. and Nyborg, K. (2003), An economic model of moral motivation, Journal of Public Economics 87, 1967-1983.

Grossman, S.J. (1981), The informational role of warranties and private disclosure about product quality, Journal of Law and Economics 24, 461-483.

Helm, D., Hepburn, C. and Mash, R. (2003), Credible carbon policy, Oxford Review of Economic Policy 19, 438-450.

Kallbekken, S., Westskog, H. and Mideksa, T.K. (2010), Appeals to social norms as policy instruments to address consumption externalities, Journal of Socio-Economics, doi:10.1016/j.socec.2010.02.007.

Kydland, F.E. and Prescott, E.C. (1977), Rules rather than discretion: The inconsistency of optimal plans, Journal of Political Economy 85, 473-492.

Milgrom, P. and Roberts, J. (1986), Relying on the information of interested parties, RAND Journal of Economics 17, 18-32.

Nyborg, K. and Rege, M. (2003), Does public policy crowd out private contributions to public goods?, Public Choice 115, 397-418.

Owen, A.L., Videras, J. and Wu, S. (2008), More information isn't always better: The case of voluntary provision of environmental quality, mimeo, Hamilton College (http://ssrn. com/abstract $=1264059)$.

Rogoff, K. (1985), The optimal degree of commitment to an intermediate monetary target, Quarterly Journal of Economics 100, 1169-1189.

Tirole, J. (1986), Hierarchies and bureaucracies: On the role of collusion in organizations, Journal of Law, Economics, and Organization 2, 181-214. 


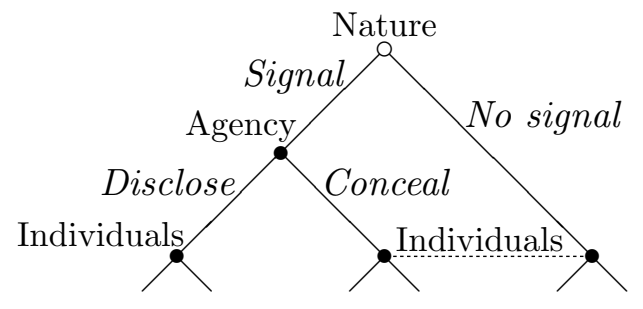

Figure 1: The game between the environmental agency and the individuals. 